\title{
Thermal orientation and thermophoresis of anisotropic colloids: The role of the internal composition ${ }^{\star}$
}

\author{
Oliver R. Gittus ${ }^{\mathrm{a}}$, Juan D. Olarte-Plata ${ }^{\mathrm{b}}$, and Fernando Bresme ${ }^{\mathrm{c}}$ \\ Department of Chemistry, Molecular Sciences Research Hub, Imperial College London, London W12 0BZ, UK
}

Received 5 March 2019 and Received in final form 7 June 2019

Published online: 18 July 2019

(C) The Author(s) 2019. This article is published with open access at Springerlink.com

\begin{abstract}
The drift motion experienced by colloids immersed in a fluid with an intrinsic temperature gradient is referred to as thermophoresis. An anisotropic mass distribution inside colloidal particles imparts a net orientation with respect to the applied thermal field, and in turn alters the thermophoretic response of the colloids. This rectification of the rotational Brownian motion is called thermal orientation. To explore the sensitivity of the thermal orientation effect with the internal composition of colloids, we investigate the thermophoretic response of rod-like colloids in the dilute regime, targeting different internal mass distributions. We derive phenomenological equations to model the dependence of the Soret coefficient with degree of mass anisotropy and test these equations using non-equilibrium molecular dynamics simulations. Using both theory and simulation, we show that the average orientation and the Soret coefficients of the colloids can depend significantly on the internal mass distribution. This observation suggests an approach to identify internal colloidal compositions using thermal gradients as sensing probes.
\end{abstract}

\section{Introduction}

Temperature gradients induce a plethora of coupling effects, allowing the non-equilibrium response of colloidal suspensions to be tuned by their microscopic properties, and for particles to be distinguished by their response. Most famously Ludwig and Soret discovered the Soret effect in aqueous solutions, showing that a thermal gradient induces a concentration gradient, a phenomenon called thermodiffusion $[1,2]$. Similar effects are observed in colloidal suspensions: thermal fields rectify the Brownian motion of colloids, which move either to hot or cold sources. This phenomenon, thermophoresis, has been a matter of intense investigation in recent years. The combination of experiments and theory have advanced this research field considerably, and it is now well established that the thermophoretic drift of colloids in solution towards cold (thermophobic) or hot (thermophilic) regions, varies with the colloid-solvent interactions, ionic strength and colloid properties, such as size, mass, moment of inertia or charge [3-7]. As such, the Soret effect has been exploited to manipulate mixtures of nanoscopic particles

\footnotetext{
* Contribution to the Topical Issue "Thermal NonEquilibrium Phenomena in Soft Matter", edited by Fernando Bresme, Velisa Vesovic, Fabrizio Croccolo, Henri Bataller.

a e-mail: o.gittus18@imperial.ac.uk

b e-mail: j.olarte@imperial.ac.uk

c e-mail: f.bresme@imperial.ac.uk
}

and biomolecules [8-13]. Notwithstanding these advances, it remains difficult to predict whether a colloid will be thermophobic or thermophilic at a specific temperature or composition of the solution.

Recently, it was shown that the internal degrees of freedom of molecules can couple with thermal fluxes, inducing a preferred orientation in a molecular fluid. It was demonstrated that water polarizes as a response to a thermal gradient [14], a phenomenon known as thermal orientation or thermal polarization, if the thermal gradient induces an electrostatic field in the fluid. The notion of thermal orientation applies to other molecular fluids, and a relationship between molecular anisotropy and orientation, as well as the Soret coefficient, was established in fluids consisting of diatomic molecules [15]. This work has motivated a number of theoretical studies focusing on thermal orientation in fluids of particles with shape and/or mass anisotropy [16-19].

The application of the thermal orientation effect to colloidal suspensions is of great interest, since this physical effect opens new avenues to manipulate anisotropic colloids in solution. The response of axially symmetric, elongated particles in a thermal gradient has been considered before in aerosols and suspensions, using kinetic theory [20] and hydrodynamic simulations [21]. It was concluded that these particles undergo anisotropic thermophoresis. Recently, it was shown that the internal composition of the particles, specifically a mass gradient, results in a thermophoretic torque [22]. The existence of 
this torque has been corroborated in both colloidal suspensions consisting of small and large colloids, relative to the characteristic diameter of the solvent molecules. For the small colloid/solvent size ratio, the existence of thermophoretic torques was observed using non-equilibrium molecular dynamics simulations [22], while for the large colloid/solvent size ratio the simulations were performed using a non-equilibrium Stochastic Rotation Dynamics approach [23]. It was also demonstrated that the orientation of the anisotropic colloids can be described using the von Mises probability distribution.

A key message from these recent works is that the orientation of a colloid in a thermal field leads to different Soret coefficients. Consequently, the Soret coefficients depend on the magnitude of the thermal gradient. The degree of orientation and the change in the magnitude of the Soret coefficient depend on a new variable, the mass dipole, which quantifies the degree of internal mass anisotropy of a colloid.

One question we wish to address in this work is whether the thermophoretic torque and the measured Soret coefficient depend significantly on the internal mass distribution inside the colloid. Ultimately, we wish to understand how the Soret coefficient of colloids with the same total mass but different mass distribution depend on the colloid internal composition. We analyse this question in the present paper using non-equilibrium molecular dynamics simulations in combination with a theoretical approach that builds on the recently discovered link between the von Mises distribution and the thermal orientation. We use this connection to derive equations that describe the dependence of the Soret coefficient with the degree of orientation of the colloids, and that allow a systematic analysis of the thermal orientation with the composition of the colloids.

\section{Theoretical background}

In this section, we derive equations to express the Soret coefficient of the colloid in terms of its internal mass distribution. (In sect. 4.3 this objective is furthered by directly linking the Soret coefficient of the colloid with its average orientation.) In our previous work [23] the interaction between the colloid and the thermal field was defined as $U(\theta)=-\left(k_{E} / 2\right) \cos \left(\theta-\theta_{0}\right)$, which is a minimum when the colloid reaches the mechanical equilibrium at $\theta_{0}$. The strength of this potential is defined by a force constant, $k_{E}$. This equation exploits an analogy between the orientation induced by a thermal field on a colloid with given mass dipole, and the orientation induced by an electrostatic field on an electrostatic dipole.

The normalized probability distribution for the colloid adopting a specific orientation with a given angle, $\theta$, with respect to the direction of the thermal field is given by the von Mises distribution on the half circle:

$$
p(\theta)=\frac{\exp (\kappa \cos \theta)}{\pi I_{0}(\kappa)},
$$

where $\kappa=k_{E} /\left(2 k_{B} T\right), I_{0}$ is the modified Bessel function of first kind and $\cos \theta=\mathbf{u}_{c} \cdot \mathbf{u}_{J_{q}}$. Unit vectors $\mathbf{u}_{c}$ and $\mathbf{u}_{J_{q}}$ define the orientation of the colloid and the direction of the heat flux, respectively.

Once the probability distribution is known, the average orientation $\langle\cos \theta\rangle$ can be obtained from

$$
\langle\cos \theta\rangle=\frac{\int_{0}^{\pi} \cos \theta \sin \theta p(\theta) \mathrm{d} \theta}{\int_{0}^{\pi} \sin \theta p(\theta) \mathrm{d} \theta},
$$

where $\sin \theta$ is the angular weighting factor which follows from the Jacobian in spherical coordinates. The solution of eq. (2) for $\theta_{0}=\pi$ is the negative of the well-known Langevin function $\mathcal{L}(\kappa)$ :

$$
\langle\cos \theta\rangle=-\mathcal{L}(\kappa)=\frac{1}{\kappa}-\operatorname{coth} \kappa .
$$

To obtain an equation for the Soret coefficient that depends on the colloid orientation, we follow the idea introduced in ref. [21], and assume a linear decomposition of the thermophoretic forces in terms of contributions parallel and perpendicular to the thermal field.

The component of the drifting velocity in the direction of heat flux, $v_{J_{q}}$, of a colloid with an orientation angle $\theta$, can be decomposed (in the body frame of reference) into parallel and perpendicular components:

$$
\begin{aligned}
& v_{\|}=v_{J_{q}} \cos \theta, \\
& v_{\perp}=v_{J_{q}} \sin \theta .
\end{aligned}
$$

The friction forces in the body frame of reference associated with $v_{J_{q}}$ can be written as

$$
\begin{aligned}
& F_{\|}=-\zeta_{\|} v_{J_{q}} \cos \theta, \\
& F_{\perp}=-\zeta_{\perp} v_{J_{q}} \sin \theta,
\end{aligned}
$$

where $\zeta_{\|}$and $\zeta_{\perp}$ are the parallel and perpendicular friction coefficients of the colloid. The friction force due to $v_{J_{q}}$, projected along the direction of heat flux, can be written as

$$
F_{J_{q}}(\theta)=F_{\|} \cos \theta+F_{\perp} \sin \theta .
$$

The friction coefficient can be written in terms of the angle between the orientation vector of the colloid and the direction of the thermal field

$$
\zeta(\theta)=\zeta_{\|} \cos ^{2} \theta+\zeta_{\perp} \sin ^{2} \theta .
$$

Using eq. (2) we obtain the average friction coefficient

$$
\begin{aligned}
\langle\zeta\rangle= & \frac{\int_{0}^{\pi} \zeta_{\|} \cos ^{2} \theta \sin \theta \exp (\kappa \cos \theta) \mathrm{d} \theta}{\int_{0}^{\pi} \sin \theta \exp (\kappa \cos \theta) \mathrm{d} \theta} \\
& +\frac{\int_{0}^{\pi} \zeta_{\perp} \sin ^{3} \theta \exp (\kappa \cos \theta) \mathrm{d} \theta}{\int_{0}^{\pi} \sin \theta \exp (\kappa \cos \theta) \mathrm{d} \theta} .
\end{aligned}
$$

Integrating eq. (10) leads to the expression

$$
\begin{aligned}
\langle\zeta\rangle= & \zeta_{\|}\left(\frac{\kappa^{2}-2 \kappa \operatorname{coth} \kappa+2}{\kappa^{2}}\right) \\
& +\zeta_{\perp}\left(\frac{2 \kappa \operatorname{coth} \kappa-2}{\kappa^{2}}\right) .
\end{aligned}
$$


It has been shown in previous work [22] that the Soret coefficient of symmetric rods (i.e. with a homogeneous mass distribution) varies with the colloid mass according to, $S_{T} \propto\left(M-m_{s}\right) /\left(M+m_{s}\right)$, where $M$ and $m_{s}$ are the mass of the colloid and the solvent, respectively. This equation is similar to the equation derived with kinetic theory for mixtures of elastic spheres interacting through $1 / r^{12}$ repulsive potentials $[24,25]$. Here, we assume that the thermal diffusion coefficient $D_{T}$ follows a similar dependence with the colloid/solvent mass ratio, giving:

$$
D_{T}=B\left(\frac{M-m_{s}}{M+m_{s}}\right)+C,
$$

where $B$ and $C$ are constants that depend linearly on the size of the colloid (see ref. [22]). It has been assumed in eq. (12) that $D_{T}$ is only weakly dependent on the orientation of the colloid. Advancing the discussion below, we find that this assumption leads to equations that accurately reproduce our simulations results.

Combining eqs. (11) and (12), and using the Sutherland-Einstein-Smoluchowski relation for the diffusion coefficient $D=k_{B} T / \zeta$, we find the following expression for the Soret coefficient in terms of the von Mises parameter $\kappa$ :

$$
\begin{aligned}
S_{T}= & \frac{1}{k_{B} T}\left[B\left(\frac{M-m_{s}}{M+m_{s}}\right)+C\right]\left[\zeta_{\perp}\left(\frac{2 \kappa \operatorname{coth} \kappa-2}{\kappa^{2}}\right)\right. \\
& \left.+\zeta_{\|}\left(\frac{\kappa^{2}-2 \kappa \operatorname{coth} \kappa+2}{\kappa^{2}}\right)\right]
\end{aligned}
$$

In sect. 4.2, the accuracy of this equation is tested against data obtained from non-equilibrium simulations.

\section{Non-equilibrium simulations}

We investigated the response of rod-like colloids with varying degrees of mass asymmetry. The colloids were modelled using the shish-kebab model, i.e. a rigid chain of tangent spheres of diameter $\sigma$. Asymmetric mass distributions were achieved by varying the position and mass $m_{a}$ of a single bead, dubbed the "asymmetric" bead, while all other beads had a mass $m$ equal to the mass of the solvent $m_{s}$. The degree of mass asymmetry in this model is quantified through the mass dipole $d \cdot \boldsymbol{u}_{c}=\boldsymbol{r}_{\text {com }}-\boldsymbol{r}_{g}$, where $\boldsymbol{r}_{\text {com }}$ is the centre of mass of the colloid and $\boldsymbol{r}_{g}$ is its geometric centre. For this particular model (see fig. 1(B)), the mass dipole of a colloid composed of $N$ particles can be written as

$$
d=a \frac{\gamma-1}{N+\gamma-1} \sigma,
$$

where $\gamma=m_{a} / m$ and $a=0,1, \ldots,(N-1) / 2$ is the position of the asymmetric bead. We show in fig. 1(A) the mass dipole as a function of the mass ratio, $\gamma$, and position, $a$, for a colloid of size $N=11$.

Boundary-driven non-equilibrium molecular dynamics simulations of a colloid suspended in a particle-based fluid at infinite dilution were carried out using the open-source
A

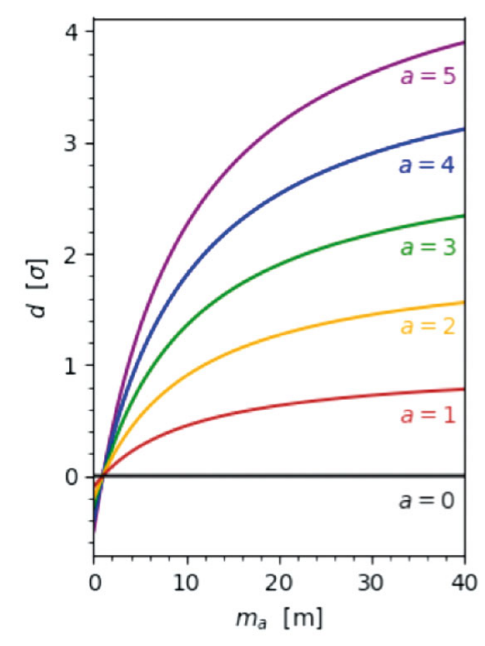

B

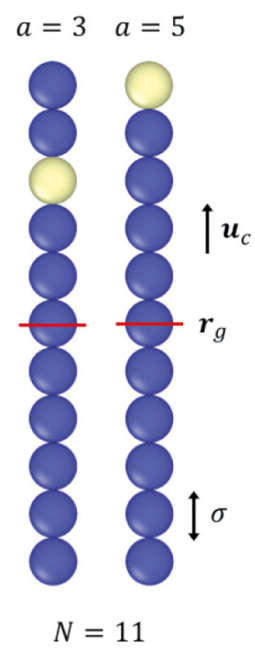

Fig. 1. (A) The range of mass dipoles $d$ for the shish-kebab model employed in this work. A colloid of length $N=11$ with a single asymmetric bead of mass $m_{a}\left(m=1 \Rightarrow m_{a}=\gamma\right)$ at position $a$ was considered. (B) Graphical representations of the colloids used in this work: rods with $a=3$ and $a=5$ are shown. The bead of mass $m_{a}$ is shown in yellow. The direction of the colloid given by the unit vector $\boldsymbol{u}_{c}$, points from the centre of geometry of the colloid, $\boldsymbol{r}_{g}$, towards the position of the asymmetric bead.

software package LAMMPS [26]. The fluid was modelled at an average density $\rho_{s}=(N / V) \sigma^{3}=1.0$ characteristic of a liquid, where $N$ and $\sigma$ are the number and diameter of solvent particles respectively, and $V=L_{x} \times L_{y} \times L_{z}=$ $(48 \times 20 \times 20) \sigma$ is the volume of the simulation cell. Thermostatting regions of size $(4 \times 20 \times 20) \sigma$ were located in the edges and centre of the simulation box (see fig. 2), such that the simulation cell is fully periodic.

A thermal gradient was established by applying the simple velocity rescaling algorithm to solvent particles in the hot and cold regions, while ensuring linear momentum conservation. The simulation set-up consists of two compartments with equal but opposite temperature gradients, and therefore equal and opposite heat fluxes. A colloid particle was placed in the centre $\boldsymbol{r}_{c}$ of each compartment, its centre of mass tethered using a harmonic potential $V(\boldsymbol{r})=k\left(\boldsymbol{r}_{\text {com }}-\boldsymbol{r}_{c}\right)^{2} / 2$, with a force constant $k \sigma^{2} / \epsilon=2 \times 10^{3}$.

Inter-particle interactions were modelled using the WCA potential:

$$
\phi_{\mathrm{WCA}}(r)= \begin{cases}4 \epsilon\left[\left(\frac{\sigma}{r}\right)^{12}-\left(\frac{\sigma}{r}\right)^{6}\right], & \text { if } r \leq 2^{1 / 6} \sigma, \\ 0, & \text { if } r>2^{1 / 6} \sigma\end{cases}
$$

where $r$ is the distance between two particles and $\epsilon$ represents the strength of the purely repulsive pair-wise interaction. Parameters $\epsilon=1$ and $\sigma=1$ were used for the colloid-fluid and fluid-fluid interactions. These parameters, $\epsilon$ and $\sigma$, are used to define the usual Lennard-Jones 
A

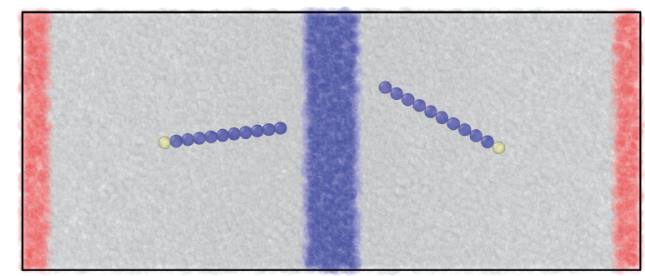

B

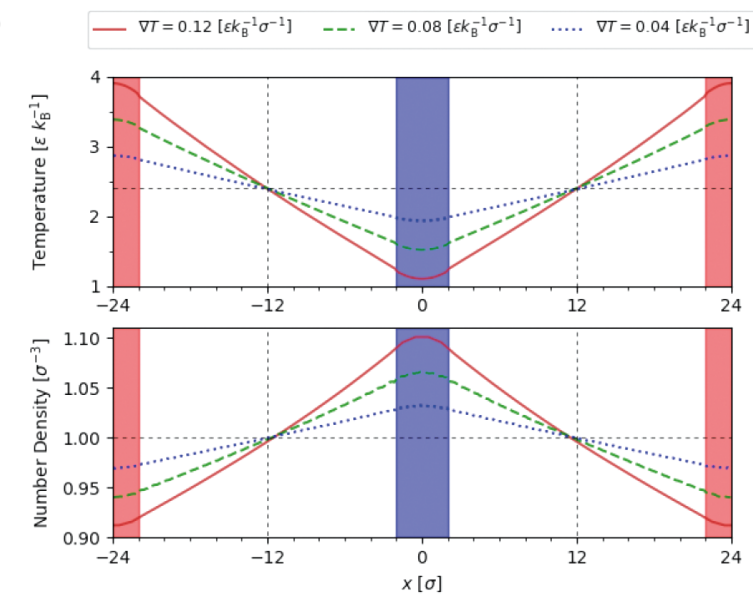

Fig. 2. (A) Snapshot of the simulation set-up, showing the thermostatting regions and asymmetric colloids with $a=5$ and $m_{a}=40$, tethered to the geometric centre of each compartment. (B) Temperature (top) and number density (bottom) profiles corresponding to systems of pure solvent with average temperature $T_{s}=2.4$ at the centre of each compartment, and $\nabla T=0.04,0.08$, and 0.12 . For large mass anisotropies, the colloid is, on average, closer to the cold thermostatting regions.

reduced units, which are adopted hereafter. The trajectories of the solvent particles were integrated using the velocity Verlet algorithm, while the translational and rotational degrees of freedom of the colloids were integrated using the method of quaternions for rigid bodies [27]. A timestep of $\delta t=0.0025 \tau$ was used for all simulations.

The orientation of the colloid, defined as $\cos \theta=$ $\mathbf{u}_{c} \cdot \mathbf{u}_{J_{q}}$, was sampled every 10 timesteps for a total of $2 \times 10^{7}$ timesteps per replica, with $20-120$ statistically independent replicas for each system (each system containing two rods). The thermophoretic force on each colloid was computed by sampling every timestep, the average displacement of the centre of mass of the colloid with respect to the restraining position, and multiplying by the force constant [28]. The Soret coefficient was then calculated from

$$
S_{T}=\frac{\boldsymbol{F}_{s}-\boldsymbol{F}_{T}}{k_{B} T \nabla T}
$$

where $\boldsymbol{F}_{s}$ and $\boldsymbol{F}_{T}$ are the thermophoretic forces on the solvent and colloid respectively (see, e.g., ref. [29]) and $T$ is the average temperature of the colloid. $\boldsymbol{F}_{s}$ was calculated by averaging over the forces experienced by particles in a system of pure solvent, using the same NEMD set-up described above.

The temperature of the colloid $T$ was measured by averaging the kinetic energy associated with each transla- tional and rotational degree of freedom of the rigid body, and is not necessarily equal to the average temperature $T_{s}$ of the solvent at the centre of the compartment, to which the centre of mass of the colloid was tethered. In our simulations, colloids with greater mass dipoles $|d|$ preferentially sample lower temperature regions.

A relevant question in our simulation approach is whether finite size effects may impact the thermal orientation and thermophoresis of the colloids. To avoid finite size effects, the dimensions of the simulation cell were chosen such that, even for the greatest mass dipole, $d$, and thermal gradient, $\nabla T$, the colloid did not enter the thermostatting region. We showed in previous work that the thermal orientation and therefore the Soret coefficients do not depend significantly on system size for simulation conditions similar to those employed here [23]. We note that this conclusion is applicable to the atomistic simulations. However, hydrodynamic simulations, e.g. based on Stochastic Rotation Dynamics (SRD), not employed here, feature a significant dependence with the size of the simulation box $[23,30,31]$. The difference in the finite size effects between the SRD and atomistic fluids can be rationalised in terms of the Schmidt number $S c=\nu / D$, which characterises the ratio of diffusive momentum transfer (through the kinematic viscosity $\nu$ ) and the diffusive mass transfer (measured through the self-diffusion coefficient $D)$. For dense fluids, such as the atomistic fluid present in this work, $S c \sim 100$. On the other hand, the mean free paths typically used in SRD lead to $S c \sim 1-10$ [32].

\section{Results and discussion}

\subsection{Thermomolecular orientation}

We performed a series of simulations using rods of fixed size $(N=11)$ but different composition: the mass of the asymmetric bead was shifted along the rod at different positions, as indicated in fig. 1. The thermal gradient induces an orientation in the colloid. We computed the average orientation of the colloids as a function of the mass $m_{a}$ and position $a$ of the asymmetric bead at an average temperature for the colloid of $T=2.4$. Figure 3 reports one of the main results of our work: colloids of the same total mass but with different internal mass distributions can exhibit very different thermal orientation responses. For a fixed mass $m_{a}$, the orientation can be increased by a factor of 4 just by shifting the position of the asymmetric bead along the axis of the colloid.

We identify three regimes for our model: one in which there is a heavy bead $(\gamma>1 \Rightarrow d>0)$ and $\langle\cos \theta\rangle<0$; the symmetric $\operatorname{rod}(\gamma=1 \Rightarrow d=0)$ for which there is no net orientation; and the case of a light bead $(\gamma<1 \Rightarrow d<0)$ and $\langle\cos \theta\rangle>0$. In the case of a heavy bead, $\langle\cos \theta\rangle<0$ indicates that in this model, the heavy bead points towards the hot region. This is consistent with the results obtained previously for the same model [22]. Simulations of diatomic molecules, and binary mixtures, indicate that the heavy component accumulates in the cold region $[15,33]$ Our result for the thermal orientation of the colloid shows 


$$
\nabla T=0.12\left[\varepsilon k_{\mathrm{B}}^{-1} \sigma^{-1}\right]
$$
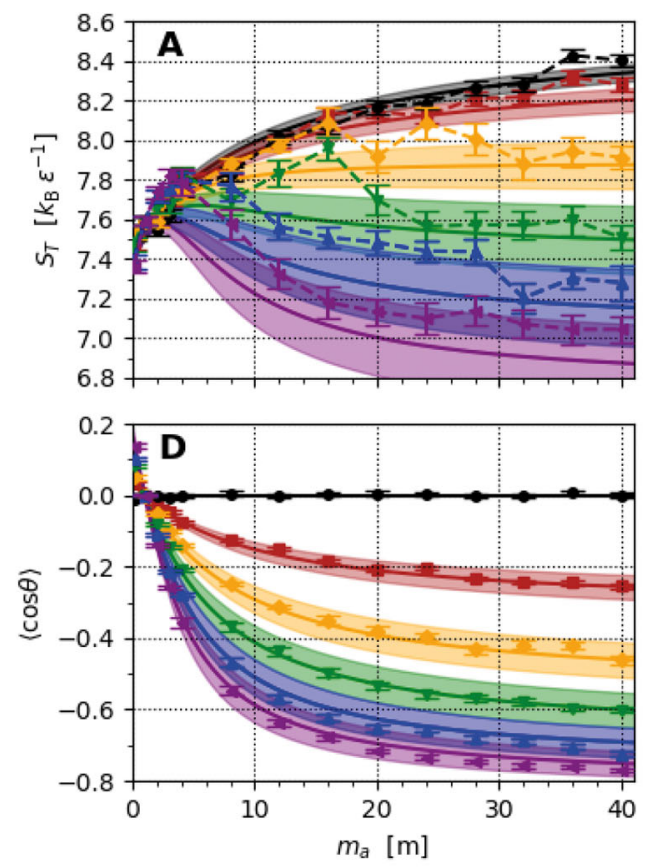

$\nabla T=0.08\left[\varepsilon k_{\mathrm{B}}^{-1} \sigma^{-1}\right]$
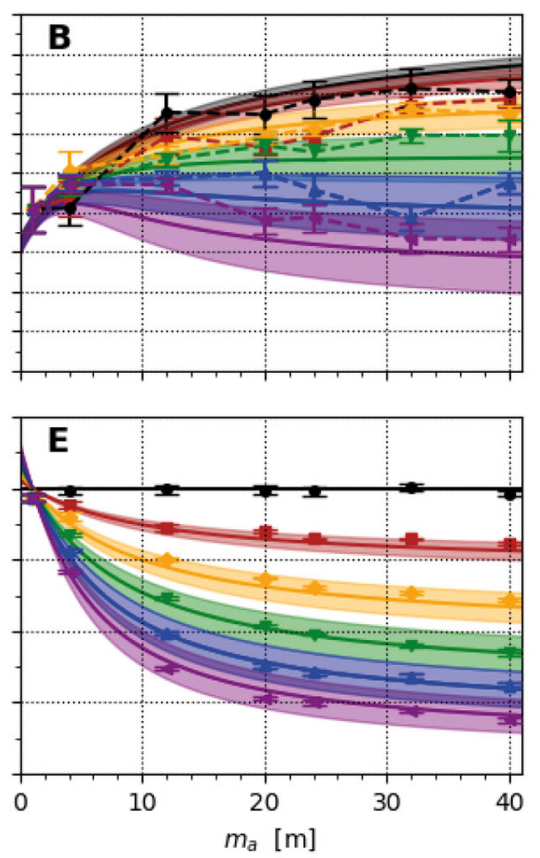

$\nabla T=0.04\left[\varepsilon k_{\mathrm{B}}^{-1} \sigma^{-1}\right]$
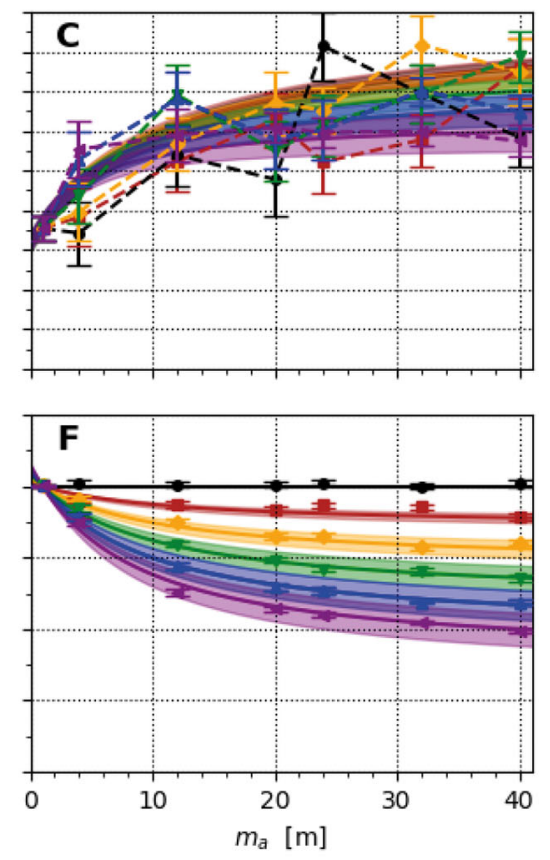

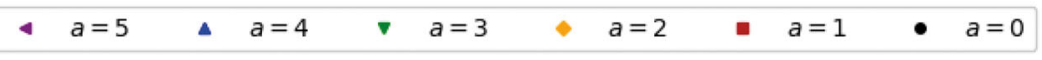

Fig. 3. Soret coefficient $S_{T}$ (top) and average orientation $\langle\cos \theta\rangle$ (bottom) of rod-like colloids (shish-kebabs) as a function of the position $a$ and mass $m_{a}$ of the asymmetric bead. From left to right: $\nabla T=0.12,0.08$ and $0.04 . a=0,1, \ldots, 5$ are shown in black, red, yellow, green, blue and purple respectively. The predictions made using phenomenological equations (eq. (3) and eq. (21), with eq. (17)) are shown by solid lines, with the shaded area representing the associated uncertainty.

that the response to the thermal gradient cannot be interpreted only in terms of the position of the heavy bead in the colloid. Clearly, the fact that the excluded volume for rotation depends on the position of center of mass, and therefore on the mass dipole, adds an extra degree of complexity, which finally determines the preference of the colloid to orient towards hot or cold regions.

As discussed in previous work (see sect. 2.2 in ref. [22]) the dependence of the orientation with the thermal gradient and the mass dipole can be modelled accurately using the von Mises distribution and eq. (3), with $\kappa$ in eq. (3) being given by the phenomenological relation

$$
\kappa=\alpha \frac{N d \nabla T}{T},
$$

where $\alpha$ is a constant that depends on the nature of the solvent. For our model, the only unknown variable in eq. (17) is $\alpha$; we determined $\alpha=1.9 \pm 0.3$, by fitting the simulation data for the smallest gradient $\nabla T=0.04$ to eq. (3) via eq. (17). This fitting includes the results for all the different dipole moments studied for the rod of size $N=11$, i.e. $a=0 \ldots 5$. We show in fig. 3(D)-(F) that the theoretical framework based on the von Mises probability distribution (eq. (3)) and the phenomenological equation (eq. (17)) accurately reproduce the dependence of the orientation with $m_{a}$ and $d$, within the uncertainty (see shadowed areas in fig. 3(F)) associated with $\alpha$, which was obtained numerically from the simulation results. In addition, our simulation results corroborate the validity of eq. (17), as shown explicitly in fig. 4.

The approach used here to obtain $\kappa$ relies only on the knowledge of the average orientation $\langle\cos \theta\rangle . \kappa$ can be extracted from the Langevin function (eq. (3)), and is related, via eq. (17), to experimental conditions ( $\nabla T$ and $T)$ as well as properties of the colloid and solvent $(N$, $d$ and $\alpha$ ). Thus, our analysis suggests an experimental route to quantify the orientation probability distribution and colloidal properties from measurements of the average orientation of the colloids. The thermophoretic torque acting on the colloids can also be obtained from $\kappa$ using this theoretical framework [23].

We tested the accuracy of eqs. (3) and (17) by calculating the orientations at two other thermal gradients, $\nabla T=0.12,0.08$. We emphasize that the theoretical curves represented in fig. 3(D), (E) are based only on data obtained at the weakest gradient (fig. $3(\mathrm{~F})$ ), and are therefore true predictions. The agreement with the simulation data is excellent; all $\langle\cos \theta\rangle$ values are within the estimated uncertainty of our predictions, which were propagated from the uncertainty of $\alpha$. Further support for the predictive power of our theoretical approach is shown in fig. 4, where we show that the general dependence of the von Mises parameter with the mass dipole can be represented in a single master curve by taking into account 


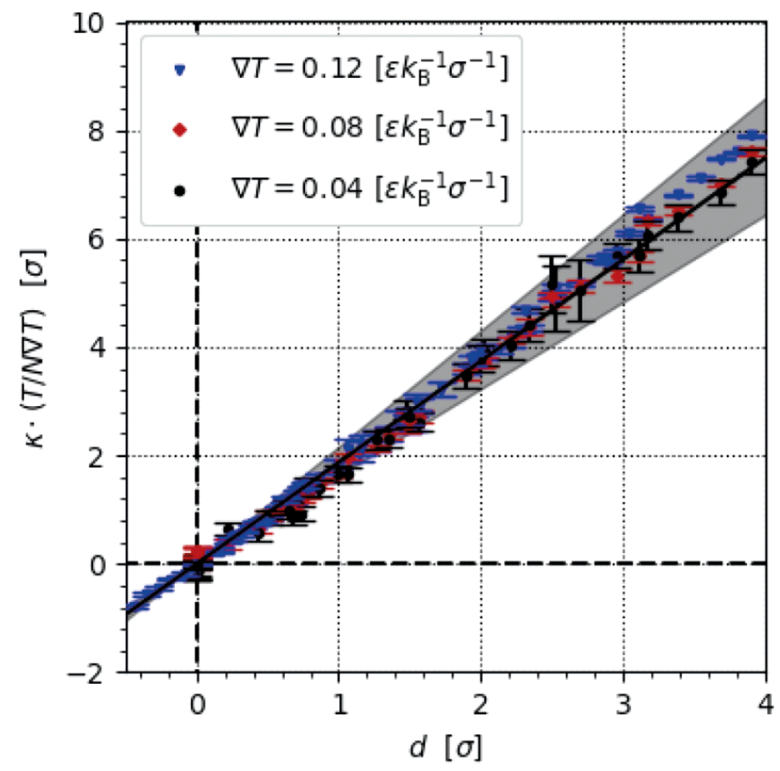

Fig. 4. The "reduced" von Mises parameter $\kappa \cdot(T / N \nabla T)$ as a function of the mass dipole $d$. The solid line represents the prediction made using the phenomenological eq. (17). The shaded area represents the associated uncertainty. As discussed in the main text the parameter $\alpha$ was fitted using the data obtained with $\nabla T=0.04$ only.

differences in the thermal gradient, $\nabla T$. The average colloid temperature, $T$, and size of the colloid, $N$, were the same in all cases investigated here.

Using eqs. (3) and (17) it is possible to plot a master curve that describes the general dependence of the average orientation of the colloids with the mass dipole (fig 5(A)). All the average orientations for different mass dipoles and for a given thermal gradient fall on the same master curves, whose slope varies with the magnitude of the thermal gradient, $\nabla T$. The dependence with $\nabla T$, can be summarized in a simple equation by taking the Taylor series expansion of the Langevin function:

$$
\begin{aligned}
& \langle\cos \theta\rangle=-\frac{\kappa}{3}+\frac{\kappa^{3}}{45}+\mathcal{O}\left(\kappa^{5}\right)+\ldots \\
& \approx-\frac{\kappa}{3}=-\frac{\alpha N d \nabla T}{3 T}, \quad|\kappa| \ll 1
\end{aligned}
$$

which applies for small $\kappa$. Noting that $N, \alpha$ and $T$ have been held constant throughout the performed simulations, $\langle\cos \theta\rangle / \nabla T$ is therefore predicted to be proportional to $d$ for $|\kappa| \ll 1$. We test this prediction in fig. 5(B); the results do indeed collapse onto a single master curve at small dipole values, $d \rightarrow 0$. Furthermore we find that the approximation for small $\kappa$ provides a good estimate of the orientation at relatively large mass dipoles, $d \sim 1$, corresponding to $\langle\cos \theta\rangle / \nabla T \sim-3$. In the context of pure fluids of anisotropic particles, linear relationships similar to eq. (19) have been derived by adopting a local equilibrium, mean-field theory approach [16-18].
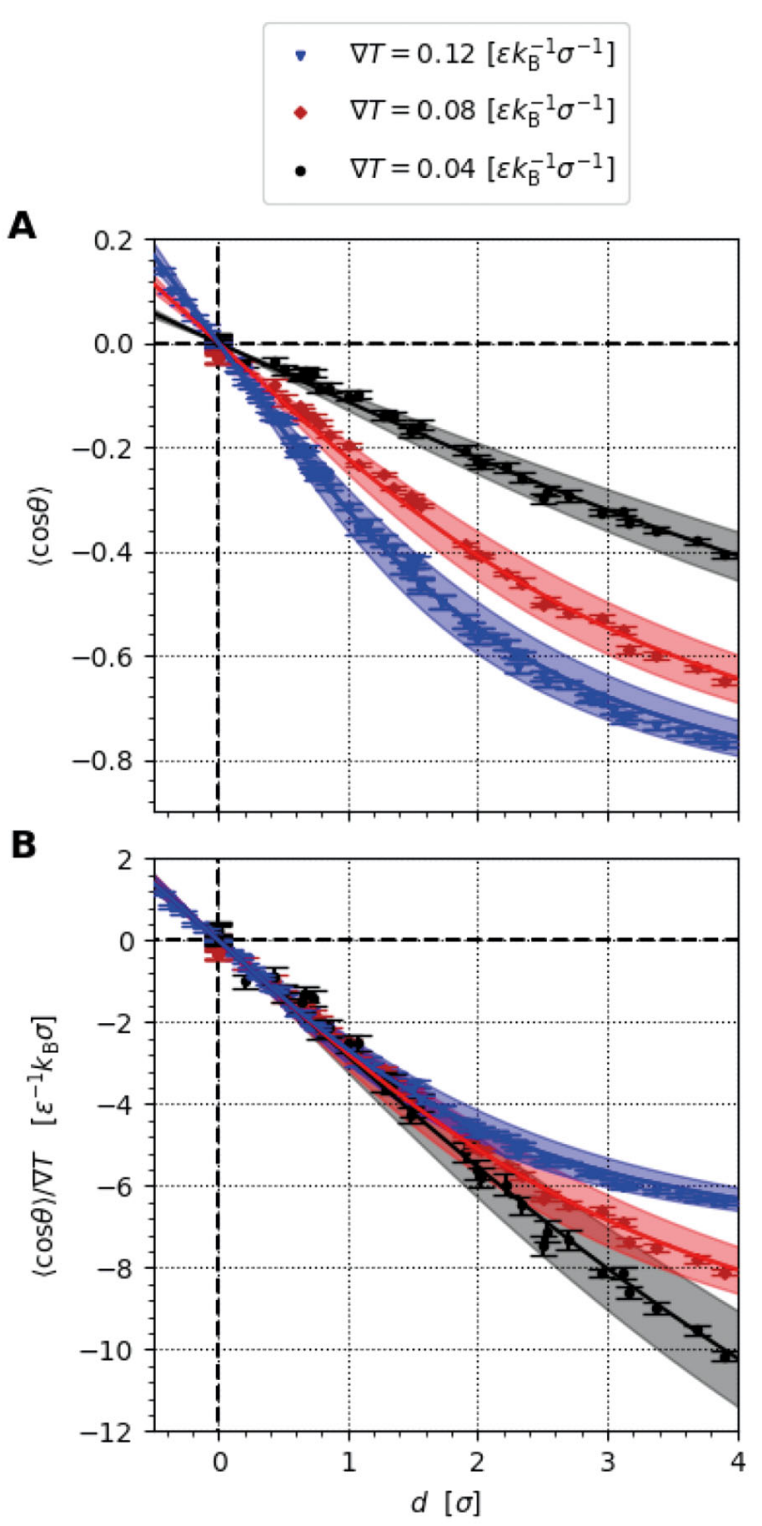

Fig. 5. Average orientation $\langle\cos \theta\rangle(\mathrm{A})$ and $\langle\cos \theta\rangle / \nabla T$ (B) as a function of mass dipole $d$ for different temperature gradients $\nabla T$. The predictions made using the phenomenological equation (eq. (3) with eq. (17)) are shown by solid lines, with the shaded area representing the associated uncertainty.

\subsection{Soret coefficient}

So far, we have established that colloids of the same total mass and different internal mass distribution feature very different thermal orientations. We discuss in the following the impact of the different thermal orientation response on the Soret coefficient. Addressing this point has particular significance, since the Soret coefficient provides a direct comparison with experimental measurements.

The Soret coefficient was computed using eq. (16). For all cases, the colloidal particle experiences a thermophoretic force in the direction of the cold region, hence the response is thermophobic and $S_{T}>0$, in agreement with previous computations for this model [22]. The 
Soret coefficients are of the order of $7-8$ in reduced units, which correspond to $0.07-0.08 \mathrm{~K}^{-1}$ using typical interaction strengths for simple fluids (i.e., $\varepsilon / k_{B}=10^{2} \mathrm{~K}^{-1}$ ). These values are intermediate between the Soret coefficients obtained typically for binary mixtures and for small colloids. In the case of the symmetric rod ( $a=0$ uniform mass distribution or zero mass dipole), we find a monotonic increase of $S_{T}$ with the total mass of the colloid, which follows the same functional form as the thermal diffusion coefficient given by eq. (12), as pointed out in ref. [22].

For colloids with mass asymmetry, the thermal orientation effect couples with its thermophoretic response (eq. (11)), resulting in a non-monotonic increase of the Soret coefficient with the total mass of the colloid. For large thermal orientation responses induced by large temperature gradients, the Soret coefficient features a maximum. The Soret coefficient of very heavy colloids can be smaller than that of lighter colloids.

We consider now the theoretical modelling of the Soret coefficient when it is coupled with the thermal orientation effect. For a colloid composed of $N$ tangent spheres of diameter $\sigma$, the parallel and perpendicular friction coefficients are given by the Kirkwood theory [34]

$$
\zeta_{\perp}=2 \zeta_{\|}=\frac{4 \pi \eta N \sigma}{\ln (N / 2)},
$$

where $\eta$ is the viscosity of the solvent. It follows from eq. (20) that the friction coefficient can be written in terms of $\zeta_{\|}$only. This allows eq. (13) to be simplified as

$$
S_{T}=\frac{D_{T} \zeta_{\|}}{k_{B} T}\left[1+\frac{2}{\kappa}\left(\operatorname{coth} \kappa-\frac{1}{\kappa}\right)\right] .
$$

The viscosity of the solvent, $\eta=4.24 \pm 0.01$, in eq. (20) was obtained using the Green-Kubo approach (see appendix B) $[35,36]$. Thus, with the aim of applying eq. (21), both $\kappa$ and the friction coefficient are known for a given colloid. We now assume that $D_{T}$ depends weakly on the orientation and use the simulation results for the Soret coefficient of the symmetric colloid $\left(m_{a}=m=1.0\right)$ to fit the constants $B$ and $C$ appearing in eq. (12). These coefficients were fitted using data corresponding to only $\nabla T=0.12$, as these provide a more accurate estimate of the Soret coefficient. We found that the parameters $B=(5.2 \pm 0.1) \times 10^{-2}$ and $C=(2.0 \pm 0.1) \times 10^{-2}$ in eq. (12) model accurately the $S_{T}$ values obtained from simulations.

Figure $3(\mathrm{~A})-(\mathrm{C})$ shows the results of the simulated Soret coefficient as a function of the mass and position of the asymmetric bead, at different temperature gradients, as well as the predictions using eq. (21). While the Soret coefficient of the symmetric colloid is independent of the thermal gradient strength, the Soret coefficients of asymmetric colloids feature a strong dependence, due to the preferential orientation of the colloid in the thermal field. This observation underlines the notion that the Soret coefficient of colloids with internal mass asymmetry depend on the temperature gradient, since the degree of orientation varies with the thermal gradient. Our results show that colloids with the same total mass, but different mass dipoles (mass asymmetry) feature different Soret coefficients (fig. 3(A)-(C)), and therefore thermophoretic forces. This result suggests that colloids with different internal composition can be differentiated based on their thermophoretic response.

The theoretical model introduced in eq. (21) predicts the main dependence of the Soret coefficients for high mass, $m_{a}$, as well as the non-monotonic dependence of $S_{T}$ with $m_{a}$, which features a maximum. The theoretical predictions lie mostly within the statistical uncertainty for $m_{a}>20$, although larger deviations between theory and simulation are observed for larger asymmetries. We attribute these deviations to the approximations made when applying eq. (21) to our simulation results (discussed in the following paragraphs), and the uncertainties associated with fitting the coefficients entering eq. (21). In particular, the uncertainty in $S_{T}$ propagated from $\alpha=1.9 \pm 0.3$ increases with $\kappa$, and therefore becomes more significant at larger mass dipoles.

There are three main sources of error associated with the application of eq. (21). Firstly, the underlying assumption that $D_{T}$ does not depend on the internal mass distribution of the colloid, only its total mass. However, the good agreement between the simulation data and the predictions made from eq. (21) support our approach of decomposing the Soret coefficient into a $D_{T}$ term that is independent of the orientation, and the diffusion coefficient $D$ term that incorporates orientation effects via $\kappa$. According to Kirkwood theory the parallel diffusion coefficient of our colloid described using the shish-kebab model is twice that of its perpendicular diffusion coefficient; $D$ is therefore strongly dependent on the orientation of the colloid.

Regarding the thermal diffusion coefficient, $D_{T}$, the dependence of this property with particle orientation was investigated by Ybarra and Rosner [20] using kinetic theory to model spherocylinders in a dilute solvent, with a Knudsen number $K n \gg 1$. Ybarra and Rosner found that the thermophoretic velocity $v_{T}$ had a maximum difference of $52 \%$, between the parallel and perpendicular orientations to the thermal field. This maximum difference corresponds to the limiting case of a sphero-cylindrical particle of infinite aspect ratio (i.e., length/radius $\rightarrow \infty$ ) and a maximal momentum accommodation coefficient of $1.0[20]$. It was also found that the shorter the length of the particle the smaller the difference in $v_{T}$. These kinetic theory studies would support a dependence of $D_{T}$ with the particle orientation, although the results correspond to conditions quite different to the one studied here, where $K n<1$. In our case taking as a reference heavy colloids and large temperature gradients, the differences between theory and simulations (see $m_{a}=40$ in fig. 3(A)), quantified in terms of a relative error, are about $1 \%$. This would suggest that the dependence of $D_{T}$ with orientation is not large in the system investigated here.

Secondly, we note that the application of Kirkwood theory (and thus eq. (20)) is valid in the continuum limit, 
while in our simulations, each colloid "bead" is the same characteristic size of a solvent particle. Thirdly, $\eta$ was calculated for a WCA fluid with an average temperature and density of $T=2.4$ and $\rho=1.0$ respectively. However, the average solvent density sampled by a given simulated colloid particle may differ from this value; in our simulation set-up, rods of different mass dipoles are expected to sample different average solvent densities. Even in the case of the greatest temperature gradient $\nabla T=0.12$, the difference in local solvent density across the colloid does not exceed $\Delta \rho_{s} \approx 0.05$, which is $5 \%$ of the average solvent density (fig. 2(B)). The effect of the density gradient on the thermophoretic response of the colloid is captured in our theory via the phemenological parameter $\alpha$. Additional work is required to establish which of these three contributions leads to the larger deviations observed for colloids with greater degrees of mass asymmetry.

We have shown in fig. 3(C) the simulated Soret coefficient for the smallest gradient, $\nabla T=0.04$, investigated in this work. The data show large fluctuations, highlighting the difficulty in obtaining precise results for the Soret coefficient when the thermal gradient is small. This is despite extensive computational effort. We were unable to obtain precise estimates for $S_{T}$ despite running at least 80 replicas for each data point; this amounts to a total of at least $8 \times 10^{6} \tau(\sim 21 \mathrm{~ns}$ in argon time using the parameters of White [37]) of sampling per data point. The extensive sampling required to resolve differences in $S_{T}$ at weak thermal gradients highlights the importance of having a theoretical approach to model the thermal orientation effect, and its impact on the thermophoretic response of colloids.

\subsection{Linking the Soret coefficient and average orientation}

Equation (21) connects the Soret coefficient with experimental variables, $D_{T}, \zeta_{\|}$and temperature. This connection can be made more explicit by expressing the equation in terms of the average orientation of the colloids, which can in principle be monitored using optical techniques [38].

We use eq. (3), to rewrite the Soret coefficient (eq. (21)) in terms of the average orientation of the colloid:

$$
S_{T}=\frac{D_{T} \zeta_{\|}}{k_{B} T}\left[1-\frac{2\langle\cos \theta\rangle}{\mathcal{L}^{-1}(-\langle\cos \theta\rangle)}\right],
$$

where $\mathcal{L}$ is again the Langevin function. The inverse Langevin function does not have an analytical expression, and approximations to $\mathcal{L}^{-1}$ are an active area of research [39]. The Puso approximation [40]

$$
\mathcal{L}^{-1}(x) \approx \frac{3 x}{1-x^{3}}
$$

for $x \in[0,1]$, has a maximum relative error of $5 \%$, and offers a favourable compromise between accuracy and complexity. Using this approximation, eq. (22) becomes

$$
S_{T}=\frac{D_{T} \zeta_{\|}}{k_{B} T}\left[\frac{5+2\langle\cos \theta\rangle^{3}}{3}\right]
$$
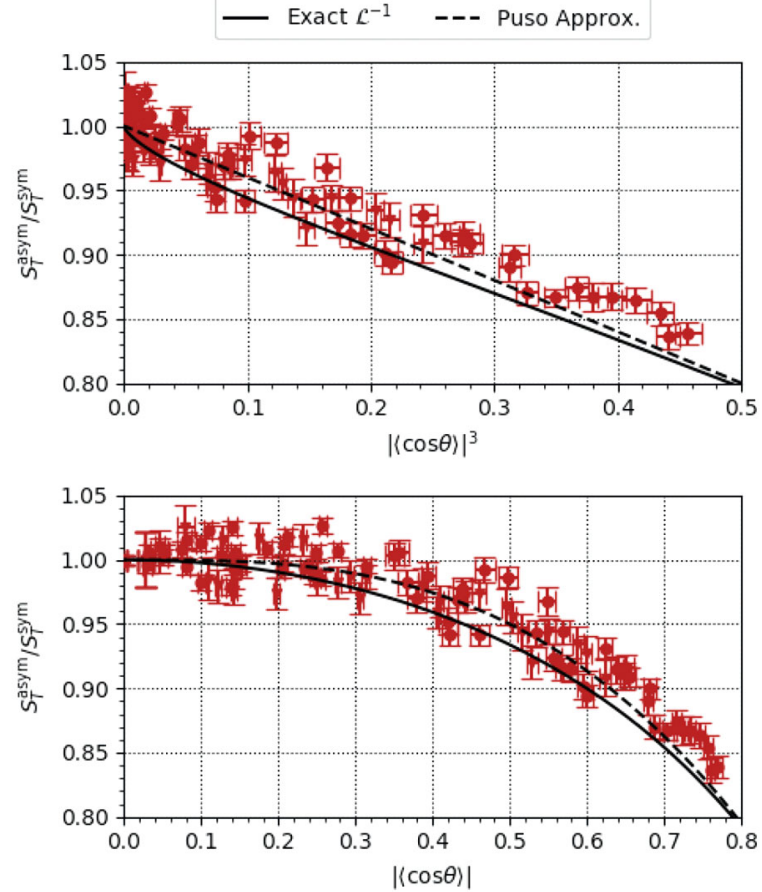

Fig. 6. Ratio of the Soret coefficient of an asymmetric $S_{T}^{a s y m}$ and symmetric $S_{T}^{s y m}$ colloids as a function of the average orientation, $\langle\cos \theta\rangle$, of the colloid in the thermal field. The theoretical predictions from eq. (22) and eq. (25) are shown by solid and dashed lines, respectively.

which now defines the Soret coefficient in terms of variables that can be fixed or measured in experiments. The change of the Soret coefficient for asymmetric colloids relative to a symmetric one of the same total mass is given by

$$
\frac{S_{T}^{\text {asym }}}{S_{T}^{\text {sym }}}=1-\frac{2}{5}|\langle\cos \theta\rangle|^{3}
$$

assuming again that $D_{T}$ does not depend on the internal mass distribution of the colloid, only on its total mass. To derive eq. (25) we have used the result of the Soret coefficient for symmetric colloids, namely, $S_{T}^{s y m}=\frac{5}{3} \frac{D_{T} \zeta_{\|}}{k_{B} T}$ (see appendix A for the derivation of this equation).

We show in fig. 6 the dependence of the ratio of Soret coefficients, asymmetric to symmetric, on the average orientation of colloids. The plot includes all our data corresponding to temperature gradients $\nabla T=0.12,0.08$, including all colloids of different compositions $\left(a, m_{a}\right)$. The data collapses onto a single master curve. We find good agreement for both the Puso approximation expressed in eq. (25), as well as the exact inverse Langevin function. This result confirms that the thermophoretic response of the colloid can be correlated to its thermal orientation, allowing the prediction of the orientation and the Soret coefficient based on the degree of asymmetry of the colloid.

It follows from eq. (25) that the Soret coefficient of the symmetric colloid is always greater than that of the asymmetric one, in other words, that a net orientation always decreases $S_{T}$. However, we find ratios $S_{T}^{\text {sym }} / S_{T}^{\text {asym }}>1$ 
based on our simulation results, even when taking into account the associated uncertainties. This can be attributed to: limited sampling, especially when trying to distinguish an asymmetric colloid with small $\kappa$ and a symmetric colloid; the underlying assumption that $D_{T}$ is independent of internal mass distribution; and the additional sources of error associated with applying eq. (21) (discussed in sect. 4.2).

\section{Conclusions}

We have investigated the thermophoretic response of colloids featuring mass anisotropy. Our work shows that an anisotropic mass distribution, quantified by the mass dipole introduced in ref. [22], biases distinctively the rotational Brownian motion of the colloid. We have shown in this work that colloids with the same total mass but different internal mass distributions adopt different average orientations, and therefore have different Soret coefficients. This orientational effect reduces the thermophoretic force experienced by the colloid. For the largest mass asymmetries $(d \approx 4)$ and thermal gradients $(\nabla T=0.12)$ explored in this study, we found that the Soret coefficient of asymmetric colloids decreases by about $20 \%$. One key message of our work is that sufficiently large mass dipoles can significantly reduce the thermophoretic force experienced by colloid particles in dilute suspensions.

To rationalize the complex coupling between the thermophoresis and thermal orientation effects, we have derived phenomenological equations to describe the dependence of these effects in terms of experimental conditions and properties of the colloid and solvent. Namely: temperature gradient, temperature, colloid geometry and mass distribution, viscosity of the solvent, and thermal diffusion coefficient. We have tested the accuracy of these equations against simulation data obtained from NEMD simulations. The good agreement between simulation and theory indicates that the average orientation and the Soret coefficient of colloids under a thermal field can be predicted from quantities such as the mass dipole and temperature gradient. Our equations further describe dependencies on additional parameters, such as colloid size, colloid shape, and the nature of the solvent, whose investigation would be a subject for future work.

One key assumption employed in the derived analytical expression for the Soret coefficient, is that the thermal diffusion coefficient $D_{T}$ is independent of the internal mass distribution of the colloid. For the system studied in this work, the orientational dependence of $D$ is expected to be more significant than for $D_{T}[20]$, and we show that incorporating orientation effects through only $D$ (via $\kappa$ ) is a good first approximation. Nevertheless, understanding the dependence of $D_{T}$ on mass asymmetry and orientation is of interest for future work, and may prove important for the description of experimental systems, consisting of colloids with complex internal compositions.

We believe that understanding the dependence of the orientation and Soret coefficient on the degree of mass anisotropy opens new avenues to manipulate dilute suspensions of asymmetric colloids. Our results indicate that the Soret coefficient is fairly sensitive to the location of a heavy "bead", or in other words to the internal mass distribution of the colloid. This variable could be exploited in applications based on thermophoresis, highlighting the importance of characterizing the degree of internal homogeneity of colloidal particles.

The next step in achieving a more comprehensive understanding of the effect of an inhomogenous mass distribution on the thermophoretic response of colloids would be experimental confirmation. We have shown that changes in $S_{T}$ can be directly linked to particle orientation effects via the orientational probability distribution for the colloid. Correlating these two experimental observables makes the validation of our approach more amenable to experimental studies, and opens a new route to characterise microscopic properties of the colloidal particles, such as their internal mass distribution.

We acknowledge the EPSRC-UK (Grant No. EP/J003859/1), the EU NanoHeal ITN project grant agreement No. 642976. ORG gratefully acknowledges a $\mathrm{PhD}$ studentship sponsored by ICL's Chemistry Doctoral Scholarship, funded by the EPSRC Doctoral Training Partnership Account. We thank the Imperial College High Performance Computing Service for providing computational resources. We wish to thank Prof. J. Miguel Rubi for many helpful discussions.

\section{Author contribution statement}

All the authors contributed to the design and implementation of the research, to the analysis of the results and to the writing of the manuscript.

Publisher's Note The EPJ Publishers remain neutral with regard to jurisdictional claims in published maps and institutional affiliations.

\section{Appendix A. The Soret coefficient of symmetric colloids}

If eq. (11) is evaluated in the limit $\kappa \rightarrow 0$ (i.e., no net orientation), we recover:

$$
\zeta_{\text {sym }}=\frac{1}{3} \zeta_{\|}+\frac{2}{3} \zeta_{\perp} .
$$

Using the result from Kirkwood theory $\left(\zeta_{\perp}=2 \zeta_{\|}\right)$, we can write:

$$
\zeta_{\text {sym }}=\frac{5}{3} \zeta_{\|}
$$

Using the Sutherland-Einstein-Smoluchowski-relation between friction and diffusion coefficients $\left(D=k_{B} T / \zeta\right)$ and the definition of the Soret coefficient, we get

$$
S_{T}^{s y m}=\frac{5}{3} \frac{D_{T} \zeta_{\|}}{k_{B} T} .
$$




\section{Appendix B. Solvent viscosity from equilibrium molecular dynamics simulations}

To compute the viscosity, $\eta$, of the solvent we used the time-correlation function of the off diagonal components of the pressure tensor, and the Green-Kubo integral formula

$$
\eta=\frac{V}{k_{B} T} \int_{0}^{\infty}\left\langle P_{\alpha \beta}\left(t_{0}\right) P_{\alpha \beta}\left(t_{0}+t\right)\right\rangle \mathrm{d} t
$$

where $V$ is the volume of the simulation box, the term in brackets \langle\rangle is the autocorrelation function of the stress tensor $P_{\alpha \beta}$, and $\alpha \neq \beta$. A correlation time of $2.5 \tau$ was used for the upper limit in the integral given above.

The simulated system consisted of $N=15625 \mathrm{WCA}$ particles in a simulation box of volume $V=(25 \times$ $25 \times 25) \sigma^{3}$, corresponding to an average density $\rho=$ $(N / V) \sigma^{3}=1.0 ; 3 \mathrm{D}$ periodic boundary conditions were applied in full. A timestep of $\delta t=0.0025 \tau$ was used. Sampling was performed in the $N V T$ ensemble at a temperature of $T=2.4 \epsilon k_{B}^{-1}$ controlled by the Nosé-Hoover chain thermostat with three chains and a time constant (damping parameter) of $0.25 \tau$. Each replica consisted of $25 \tau$ of equilibration followed by a $8.72 \times 10^{5} \tau$ production run. The viscosity $\eta$ was calculated using two statistically independent replicas. We exploited the symmetry of the pressure tensor in our system to calculate the viscosity, by averaging the results obtained from the $x y, x z$ and $y z$ components of the pressure tensor.

Open Access This is an open access article distributed under the terms of the Creative Commons Attribution License (http://creativecommons.org/licenses/by/4.0), which permits unrestricted use, distribution, and reproduction in any medium, provided the original work is properly cited.

\section{References}

1. C. Ludwig, Diffusion zwischen ungleich erwärmten Orten gleich zusammengesetzter Lösung, Sitzber. Akad. Wiss. Wien, Math.-Naturwiss. Kl. 20, 539 (1856).

2. C. Soret, Arch. Sci. Phys. Nat. 3, 48 (1879).

3. A. Würger, Rep. Prog. Phys. 73, 126601 (2010).

4. R. Piazza, A. Parola, J. Phys.: Condens. Matter 20, 153102 (2008).

5. S. Wiegand, J. Phys.: Condens. Matter 16, R357 (2004).

6. C. Debuschewitz, W. Köhler, Phys. Rev. Lett. 87, 055901 (2001).

7. P.-A. Artola, B. Rousseau, Phys. Rev. Lett. 98, 125901 (2007).

8. S. Duhr, D. Braun, Proc. Natl. Acad. Sci. 103, 19678 (2006).
9. C.J. Wienken, P. Baaske, U. Rothbauer, D. Braun, S. Duhr, Nat. Commun. 1, 100 (2010).

10. Y.T. Maeda, T. Tlusty, A. Libchaber, Proc. Natl. Acad. Sci. U.S.A. 109, 17972 (2012).

11. F. Huang, P. Chakraborty, C.C. Lundstrom, C. Holmden, J.J.G. Glessner, S.W. Kieffer, C.E. Lesher, Nature 464, 396 (2010).

12. F.M. Richter, E.B. Watson, R.A. Mendybaev, F.-Z. Teng, P.E. Janney, Geochim. Cosmochim. Acta 72, 206 (2008).

13. A. Lervik, F. Bresme, Phys. Chem. Chem. Phys. 16, 13279 (2014).

14. F. Bresme, A. Lervik, D. Bedeaux, S. Kjelstrup, Phys. Rev. Lett. 101, 020602 (2008).

15. F. Römer, F. Bresme, J. Muscatello, D. Bedeaux, J.M. Rubí, Phys. Rev. Lett. 108, 105901 (2012).

16. A.A. Lee, Soft Matter 12, 8661 (2016).

17. P. Wirnsberger, C. Dellago, D. Frenkel, A. Reinhardt, Phys. Rev. Lett. 120, 226001 (2018).

18. A. Gardin, A. Ferrarini, Phys. Chem. Chem. Phys. 21, 104 (2019).

19. R.L. Jack, P. Wirnsberger, A. Reinhardt, J. Chem. Phys. 150, 134501 (2019).

20. P. Garcia-Ybarra, D.E. Rosner, AIChE J. 35, 139 (1989).

21. Z. Tan, M. Yang, M. Ripoll, Soft Matter 13, 7283 (2017).

22. J. Olarte-Plata, J.M. Rubi, F. Bresme, Phys. Rev. E 97, 052607 (2018).

23. J.D. Olarte-Plata, F. Bresme, Phys. Chem. Chem. Phys. 21, 1131 (2019).

24. W.H. Furry, R.C. Jones, L. Onsager, Phys. Rev. 55, 1083 (1939).

25. R.C. Jones, W.H. Furry, Rev. Mod. Phys. 18, 151 (1946).

26. S. Plimpton, J. Comput. Phys. 117, 1 (1995).

27. T.F. Miller, M. Eleftheriou, P. Pattnaik, A. Ndirango, D. Newns, G.J. Martyna, J. Chem. Phys. 116, 8649 (2002).

28. G. Galliero, S. Volz, J. Chem. Phys. 128, 064505 (2008).

29. M. Yang, M. Ripoll, J. Phys.: Condens. Matter 24, 195101 (2012).

30. D. Lüsebrink, M. Yang, M. Ripoll, J. Phys.: Condens. Matter 24, 284132 (2012).

31. J. Burelbach, D.B. Brückner, D. Frenkel, E. Eiser, Soft Matter 14, 7446 (2018).

32. J.T. Padding, A.A. Louis, Phys. Rev. E 74, 031402 (2006).

33. D. Reith, F. Müller-Plathe, J. Chem. Phys. 112, 2436 (2000).

34. M. Doi, S.F. Edwards. The Theory of Polymer Dynamics (Clarendon Press, 1986).

35. M.S. Green, J. Chem. Phys. 22, 398 (1954).

36. R. Kubo, J. Phys. Soc. Jpn. 12, 570 (1957).

37. J.A. White, J. Chem. Phys. 111, 9352 (1999).

38. P.V. Ruijgrok, N.R. Verhart, P. Zijlstra, A.L. Tchebotareva, M. Orrit, Phys. Rev. Lett. 107, 037401 (2011).

39. R. Jedynak, Rheol. Acta 54, 29 (2015).

40. M. Puso, Mechanistic constitutive models for rubber elasticity and viscoelasticity, $\mathrm{PhD}$ Thesis, University of California, Davis, 2003. 